\title{
Análise bibliométrica sobre a produção científica no campo das habilidades sociais
}

\author{
Eliane Colepícolo \\ Universidade Federal de São Carlos - UFSCar, Brasil
}

ANALYSIS

\begin{abstract}
Resumo
Objetivo. Esta pesquisa consistiu em contribuir com a sistematização do campo teórico-prático das Habilidades Sociais, aqui denominado Campo das Habilidades Sociais $(\mathrm{CHS})$. Método. Foi realizado o levantamento de uma amostra significativa de metadados de publicações do CHS extraídos de 15 bases de dados de literatura técnico-científica em Psicologia, disponíveis no Portal de Periódicos da CAPES. Após tratamento e refinamento, esta amostra resultou em um corpus de 25.409 metadados de artigos científicos, que é um conjunto de dados das publicações, provenientes de campos como autor, título, assunto, resumo, entre outros. Os metadados do corpus, armazenados em um banco de dados, possibilitaram a elaboração de uma série de cruzamentos de dados, resultando em indicadores bibliométricos. Resultados. Como resultados, encontram-se: 1) o Corpus HS, publicado na internet, que possibilita a inclusão de novos metadados e a recuperação da quase totalidade de artigos científicos do campo; 2) os indicadores bibliométricos em CHS, que podem oferecer subsídios ao desenvolvimento e expansão do campo. Conclusões. Os indicadores bibliométricos do CHS mostram um crescimento representativo da produção sobre habilidades sociais na década de 1970. A pesquisa sobre HS é conduzida principalmente por pesquisadores dos Estados Unidos, Brasil, Reino Unido e Dinamarca. Os principais assuntos estudados são "social skills", "interpersonal relations", "social competence", "empathy", "assertividade", "assertiveness", "children", "adulto", "college students"; e os principais periódicos são: Journal of Personality and Social Psychology; Psychological Reports; Journal of Autism and Developmental Disorders e Child Development.
\end{abstract}

Palavras-chave

Bibliometria ; Indicadores bibliométricos ; Habilidades sociais ; Campo das habilidades sociais

\section{Bibliometric analysis about the scientific production on the social skills field}

\begin{abstract}
Objective. This research consisted to contribute to the systematization of the theoretical and practical field of Social Skills, here called Social Skills Field (SSF). Method. A survey of a representative sample of CHS publications metadata extracted from the 15 scientific and technical literature databases in Psychology available in the CAPES Journals Portal was conducted. After treatment and refinement, this sample resulted in a corpus of metadata 25,409 scientific articles, which is a set of metadata publications, from fields such as author, title, subject, abstract, among others. The corpus of metadata stored in a database allowed the creation of a series of data crossing, resulting in bibliometric indicators. Results. As a result, we have: 1) the SS Corpus, published on the Internet, which enables the inclusion of new metadata and the recovery of almost all scientific articles of the field; 2) bibliometric indicators in CHS, which may offer subsidies for the development and expansion of the field. Conclusions. Bibliometric indicators of the CHS show a representative production growth of social skills in the 1970s. Research on SS is mainly conducted by researchers from the United States, Brazil, United Kingdon and Denmark. The main subject studied are "social skills", "interpersonal relations", "social competence", "empathy", "assertividade", "assertiveness", "children", "adult", "college students"; and the major journals are: Journal of Personality and Social Psychology; Psychological Reports; Journal of Autism and Developmental Disorders and Child Development.
\end{abstract}

Keywords

Bibliometrics ; Bibliometric indicators ; Social skills ; Social skills field 


\section{Introdução}

Há um interesse cada vez maior dos governos, autoridades em Ciência e Tecnologia (C\&T) e instituições de pesquisa em avaliar áreas do conhecimento e campos de pesquisa, seja para investimentos ou para destinação de recursos de fomento, utilizando indicadores que os auxiliem na tomada de decisões quanto às áreas de pesquisa com maiores necessidades de suporte financeiro e humano (Silva \& Bianchi, 2001). Com isto, vêm se estabelecendo diversos métodos e técnicas para avaliação das ciências, que podem oferecer importantes contribuições à análise panorâmica de um campo, por meio da obtenção e uso de indicadores relativos ao seu processo de desenvolvimento (Chaparro, 1996; Mugnaini, 2006, p. 83). Com isto, surgem campos de aplicação como a Cientometria e a Bibliometria, cada uma com suas especificidades.

A Cientometria consiste na aplicação de técnicas numéricas analíticas para estudar a ciência da ciência (Silva \& Bianchi, 2001), por meio de indicadores, com vistas a garantir sua validade e facilitar sua compreensão. Inclui como instrumentos de análise das publicações, os indicadores de insumo e de produto, buscando associar causas e efeitos dentro do sistema científico. É usada também como recurso para avaliar e nortear o desenvolvimento das políticas científicas (Mugnaini, 2006; Spinak, 1998).

A Bibliometria consiste no tratamento e análise estatística de um corpus documental de publicações científicas, com objetivo de produzir indicadores da produção científica e tecnológica de áreas do conhecimento e campos de pesquisa, que auxiliem na gestão e na tomada de decisão da pesquisa em C\&T. A Bibliometria pode ser considerada como campo específico da Cientometria e vem sendo utilizada por diversas áreas do conhecimento para avaliação da produção científica e do estado da C\&T em todo o mundo (Fumo, Manolio, Bello, \& Hayashi, 2009; Mugnaini, 2006, p. 86; Silva \& Bianchi, 2001).

Os indicadores bibliométricos se baseiam na premissa de que as publicações científicas denotam a presença e a qualidade das atividades de pesquisa, pois novos conhecimentos ou descobertas só adquirem valor quando difundidos na comunidade científica, por meio de publicações, que são uma etapa essencial do processo de investigação, possibilitando ao cientista o reconhecimento de seu próprio trabalho e a continuidade da pesquisa (Silva \& Bianchi, 2001).

Segundo Costa, Lopes \& Fernández-Limós (2012), indicadores bibliométricos são ferramentas de avaliação e podem ser divididos em indicadores de: 1) qualidade científica, baseados na percepção ou opinião dos pares que avaliam publicações pelos conteúdos; 2) atividade científica, que possibilitam contabilizar a produtividade dos autores e a quantidade e distribuição dos trabalhos publicados e das referências entre trabalhos e autores; 3) impacto científico, que medem o impacto dos trabalhos e das fontes de informação; e 4) associações temáticas, tais como a análise de citações e a análise de referências. Entre os principais indicadores bibliométricos encontram-se: fator de impacto, índice de citações, índice $\mathrm{H}$, métricas Eigenfactor, indicador SCImago Journal Rank (SJR) e as ferramentas bibliométricas disponíveis em diversas bases de dados, tais como a Web of Science, o Journal Scitation Reports (JCR), Scopus, Google Scholar, entre outros. Neste trabalho, o foco foi nos indicadores de atividade científica.

Para Peña (2012, p. 2), a Bibliometria pode ser considerada como um dos estudos métricos de informação mais sólido dentro do mundo informacional, resultando em um método confiável e universal para medir a produtividade de áreas de conhecimento e para obtenção de resultados confiáveis para a tomada de decisão.

Em síntese, a Cientometria avalia de forma abrangente a C\&T, por meio de todos os tipos de indicadores, seja de insumos, produtos, projetos, produção científica, inventos, patentes, enquanto a Bibliometria analisa a C\&T por meio de indicadores construídos especificamente a partir de publicações científicas, ambas auxiliando na tomada de decisão e gestão da pesquisa científica.

O foco desta pesquisa é a Bibliometria e a produção de indicadores bibliométricos, que vêm sendo utilizados como subsídio ao planejamento e gestão da C\&T. Esses indicadores podem ser usados para estudos panorâmicos sobre áreas e campos de pesquisa que venham a contribuir para sua consistência e coerência, pois oferecem o potencial de melhoria das condições teóricas e metodológicas de pesquisa e aplicação, sugerindo novos enfoques de pesquisa ou a retomada daqueles abandonados, abrindo possibilidades de ação e intervenção da comunidade científica do campo em temáticas não exploradas.

O foco da análise bibliométrica desta pesquisa foi o Campo das Habilidades Sociais (CHS), que pode ser considerado como um campo de pesquisa e aplicação do conhecimento psicológico (Bolsoni-Silva et al., 2006), relativo ao desempenho social (Z. A. P. Del Prette \& Del Prette, 1999, p. 24). 
O CHS ganhou impulso aproximadamente na década de 1970, pautado no movimento do Treinamento de Habilidades Sociais (THS), ocorrido na Inglaterra, e no movimento do Treinamento Assertivo (TA), nos Estados Unidos (Z. A. P. Del Prette et al., 2000). O CHS absorveu elementos de diversos enfoques da Psicologia, tais como do Behaviorismo e da Análise do Comportamento de Skinner (1953), da Teoria da Percepção Social de Argyle (1978) e da Teoria Social Cognitiva de Bandura (1986), entre outras. Os conceitos e teorias relacionados ao CHS surgiram e se desenvolveram em parte como forma de explicar o comportamento humano nas relações interpessoais, mas também como forma de solucionar problemas nas interações sociais, tanto em contextos sociais saudáveis, como família, escola, universidade e trabalho, quanto em contextos do cuidado em saúde, tais como hospitais psiquiátricos e clínicas, na forma de terapia comportamental.

A expansão do campo foi rápida e profícua, mas não tão bem sistematizada devido à diversidade de enfoques teóricos, surgindo diversas vertentes de modelos, teorias, terapias e aplicações em HS, o que, por um lado, promove a discussão de ideias, mas por outro, pode também promover conflitos, ambiguidade conceitual, dificuldade em se atestar a validade de instrumentos usados no campo, entre outras. Assim, cerca de 20 anos após o surgimento do CHS, muitos pesquisadores do campo já cogitavam a necessidade de sua sistematização como campo de pesquisa. Para pesquisadores como McFall (1982), Hargie, Saunders e Dickson (1994) e Trower (1995), o CHS precisa de uma teoria que reveja os diferentes construtos explicativos e que os integre de forma sistemática, de modo a melhor articular os fenômenos a que se refere, tais como habilidades sociais, interação social e comunicação interpessoal (Del Prette \& Del Prette, 2010, p. 39).

Atualmente, o CHS apresenta uma configuração dinâmica, devido a uma grande variedade de definições (Almir Del Prette, 1998), de enfoques teóricos sobre aprendizagem e desenvolvimento, os quais contribuíram para uma série de intervenções utilizadas em programas de habilidades sociais (A. Del Prette \& Del Prette, 2011, p. 26). em constante desenvolvimento e expansão, de modo que uma análise bibliométrica do campo pode ajudar a compreender seu passado, presente e tendências futuras.

São considerados como os principais conceitos e objetos de estudo e trabalho do CHS o desempenho social, habilidades sociais, treinamento em habilidades sociais e competência social (Z. A. P. Del Prette \& Del Prette, 1999, Capítulo 4). Pelo que se observa na literatura clássica sobre habilidades sociais (Argyle, 1978; Caballo, 2005; A. Del Prette \& Del Prette, 2011; Z. A. P. Del Prette \& Del Prette, 1999; Gresham, 2009), entre os objetivos do campo estão os de identificar, compreender, caracterizar, analisar, melhorar e avaliar o desempenho social, as habilidades sociais e a competência social de indivíduos e grupos.

Os objetivos específicos do CHS são: 1) estudar o desempenho social de indivíduos e grupos, identificar e caracterizar HS, déficits de habilidades sociais e competências sociais; 2) desenvolver, melhorar e avaliar instrumentos para identificação de déficits, habilidades e competências sociais, tais como escalas e inventários; 3) planejar, desenvolver e oferecer treinamentos em habilidades sociais, visando a competência social de indivíduos e grupos em interação social de qualquer espécie; 4) analisar o desempenho, as habilidades e a competência social por meio de instrumentos de avaliação, tais como escalas.

Os resultados, produtos e serviços provenientes do CHS podem ser aplicados em diversos contextos e situações, por quaisquer indivíduos e grupos que se desempenhem socialmente. É aplicável em qualquer ambiente onde haja interação social: lar, família, escola, igreja, trabalho, eventos, locais de lazer, locais de prática de esporte, entre outros. O CHS ajuda a entender, sistematizar e avaliar as interações sociais entre indivíduos e grupos, oferecendo soluções para a promoção do desenvolvimento de habilidades sociais, o bom desempenho social e a competência social.

Dada a importância do CHS, o objetivo deste estudo foi realizar a análise bibliométrica do campo, com base na produção científica contida em 25.409 artigos de periódicos sem duplicações, publicados entre 1912 e 2014 , extraídos de 15 bases de dados de literatura técnico-científica disponíveis no Portal de Periódicos da CAPES.

\section{Materiais e métodos}

Esta análise bibliométrica surgiu como parte de uma pesquisa de doutorado sobre a Cientometria do CHS, desenvolvido no Programa de Pós-graduação em Psicologia (PPGPsi) na Universidade Federal de São Carlos (UFSCar). A ideia da pesquisa foi oferecer à comunidade de pesquisadores do CHS um conjunto de indicadores, entre eles, os bibliométricos, que os auxiliem na sistematização do campo. A construção dos indicadores foi pautada no uso de recursos integrados da Ciência da Informação e Ciência da Computação. 
Inicialmente, foram realizados diversos levantamentos bibliográficos em bibliotecas e repositórios nacionais e internacionais, para revisão da literatura sobre os diversos aspectos do CHS: teorias, métodos, técnicas, aplicações e avaliação. O objetivo principal destas revisões foi a compreensão panorâmica do campo.

O processo para a análise bibliométrica envolveu duas etapas, descritas a seguir: (1) extração e tratamento de um Corpus sobre HS; (2) desenvolvimento de indicadores bibliométricos. Diversas ferramentas tecnológicas foram utilizadas durante o processo de desenvolvimento dos indicadores, principalmente o banco de dados MySQL, a linguagem de programação PHP e o framework Joomla!.

\section{1 Extração e tratamento do Corpus HS}

O corpus de análise para esta pesquisa foi obtido a partir da produção científica de Psicologia extraída de 15 portais ou bases de dados de literatura técnico-científica disponíveis no Portal de Periódicos da CAPES (2010). São elas: Academic OneFile; BVS Regional; Cambridge Journals On-line; Ebsco-Host; Journals@Ovid Full Text; JSTOR Arts \& Sciences I Collection (Humanities); Oxford Journals; portal Proquest; PsycNet; NCBI; SAGE Journals On-line; ScienceDirect; Scopus; Web of Science; Wiley Online Library. Estas bases são portais internacionais mantidos por grandes editoras científicas que indexam uma série de publicações de cunho científico e tecnológico em todas as áreas do conhecimento, incluindo Psicologia. Todas elas apresentam uma maioria de publicações no idioma inglês, que foi o idioma selecionado para composição do corpus. Publicações em outros idiomas também foram recuperadas, desde que apresentassem título e resumo em inglês, inclusive a literatura latino-americana e brasileira.

A maioria destas bases utiliza como critérios para aceite de publicações a revisão pelos pares, periodicidade regular, heterogeneidade de corpo editorial como forma de garantir a qualidade e confiabilidade destas. Estas bases geralmente apresentam indicadores bibliométricos, tais como fator de impacto, índice de citações, entre outros. Ainda que haja controvérsias quanto à validade destes indicadores, atualmente são os que mais sustentam a qualidade e confiabilidade das publicações.

A estratégia de busca padrão para todas as bases envolveu os termos-chave "social skills", "social competence" e "assertivity" presentes nos campos de título (title) e assunto (subject); entretanto, as estratégias foram customizadas para cada base, devido às diferentes nuances nos seus mecanismos de buscas. Os termos-chave foram determinados por docentes especialistas no CHS, considerados como os termos mais representativos do campo.

A extração dos metadados foi realizada entre 2012 e 2013, utilizando técnicas de exportação de registros por XML e exportação de referências bibliográficas usando o software livre RefBase, que oferece opção de exportação a partir de diversos formatos de referência bibliográfica diretamente para uma base em MySQL. Foram obtidos aproximadamente 104.000 registros a partir da coleta inicial.

Após a inclusão de todos os registros no MySQL, que é um sistema de banco de dados relacional, iniciou-se o tratamento dos metadados para padronização e exclusão de registros duplicados. Após o tratamento do corpus, os metadados foram analisados por especialistas do CHS, concluindo-se que muitas publicações eram de outras áreas; por isso, foi feito o refinamento com uma estratégia mais específica, limitada ao tipo de documento "artigo científico" (article) e aos termos-chave "social skills", "social competence" e "assertivity" presentes nos campos de título (title) e assunto (subject), reduzindo o corpus para 25.409 artigos. O Corpus HS, tratado e refinado encontra-se disponível on-line, em um site desenvolvido em framework Joomla!, baseado em MySQL, PHP e HTML, que possibilita a inclusão, exclusão, pesquisa e visualização dos metadados de forma dinâmica.

\subsection{Desenvolvimento de indicadores bibliométricos}

Os indicadores bibliométricos de atividade científica são resultados estatísticos de frequência de determinadas atributos das publicações, tais como autor, assunto, periódico e ano de publicação. Foram obtidos por meio de consultas SQL, usando técnicas de agrupamento e classificação de dados em banco de dados relacionais, que resultaram na sumarização de informações e na composição de estatísticas. As contagens e porcentagens dos indicadores bibliométricos são baseadas no total de publicações que relaciona HS a cada um dos resultados apresentados. Com os indicadores calculados, foram realizadas inferências relativas aos resultados obtidos, os quais foram sintetizados em tabelas e gráficos e são apresentados a seguir. 


\section{Resultados e discussão}

Os principais resultados obtidos são os indicadores bibliométricos em HS, baseados no Corpus HS, contendo frequências e rankings de publicações, os quais foram disponibilizados, desde o inicio de 2014, no site "Estudo Cientométrico das Habilidades Sociais" (http://www.phs.rihs.ufscar.br). Estes indicadores bibliométricos são dinâmicos, pois, na parte administrativa deste site, é possível incluir metadados de novas publicações que forem surgindo, o que altera os valores dos indicadores a cada novo registro inserido no Corpus HS. O site encontrase sob a coordenação do Laboratório de Interação Social (LIS) do PPGPsi/UFSCar para manutenção e inclusão de novos registros. Nesta pesquisa, são apresentados os resultados iniciais com base nos 25.409 registros do Corpus HS, sem inclusão de novos metadados. Os indicadores bibliométricos apresentados são: total de publicações sobre HS por décadas e por ano, além dos rankings de publicações por autor, por assunto e por periódico.

\subsection{Evolução periódica das publicações em HS}

Os indicadores bibliométricos de publicações em HS ao ano e por décadas (Figura 1) mostram um grande salto de publicações sobre HS a partir da década de 1970, que é considerado como o período com maior expansão na década de 2000 e tendência a um crescimento exponencial, já que a soma dos totais dos últimos quatro anos (2010 a 2013) representam mais de 50\% da década anterior.

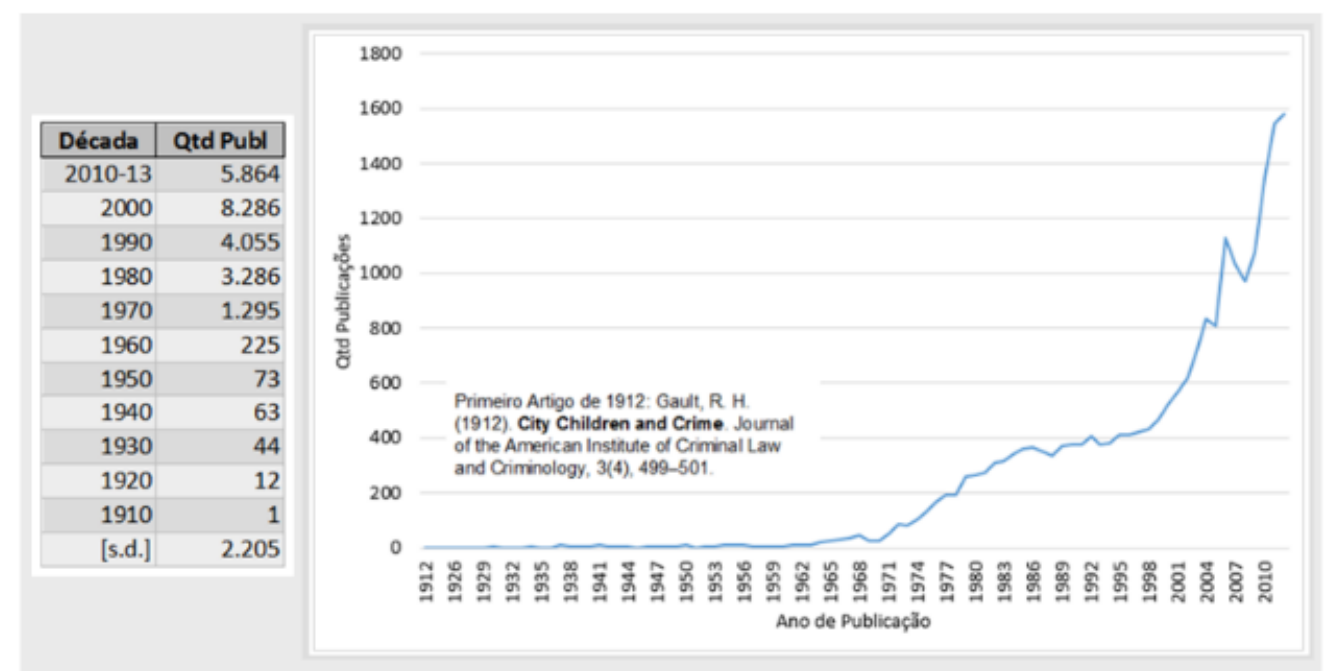

Figura 1: Evolução da produção científica de artigos sobre Habilidades Sociais ao Ano e por Décadas, ao longo do período de 1912 a 2014.

Fonte: A autora (2014)

A título de curiosidade, o primeiro artigo publicado que tem relação com o CHS trata dos problemas de ajustamento social, delinquência juvenil, desemprego e criminalidade na cidade de Chicago (Gault, 1912), o qual apresentou em suas palavras-chave o termo "social skills". Há uma quantidade considerável de artigos não datados ([s.d.]), o que pode indicar que nem sempre os dados das publicações nas bases estão completos, por isso é importante que o corpus fique disponível para atualização posterior.

De modo geral, os dados de evolução ao longo do tempo do CHS apresentam uma perspectiva de crescimento, expansão e, consequentemente, de maior especialização quanto aos temas abordados pelo campo.

\subsection{Publicações em HS por Autor}

Os indicadores bibliométricos de publicações em HS por autor ( Tabela 1) apresentam uma grande quantidade de autores provenientes dos Estados Unidos, tais como Frank M. Gresham, Johnny L. Matson, Nancy Eisenberg, Allan S. Belack, entre outros. 
Tabela 1 : Ranking de Autores com mais de 20 artigos sobre Habilidades Sociais no período de 1912 a 2014.

\begin{tabular}{|l|l|r|}
\hline \multicolumn{1}{|c|}{ Autor } & Pais & QtdP ub \\
\hline Gresham Frank M & US & 78 \\
\hline Matson Johnny L & US & 75 \\
\hline Hersen Michel & US & 60 \\
\hline Eisenberg Nancy & US & 54 \\
\hline Bellack Alan S & US & 52 \\
\hline Mueser Kim T & US & 46 \\
\hline Elliott Stephen N & US & 38 \\
\hline Del Prette ZAP & BR & 38 \\
\hline Bond GR & US & 37 \\
\hline Strain Phillip S & US & 35 \\
\hline
\end{tabular}

\begin{tabular}{|l|l|r|}
\hline \multicolumn{1}{|c|}{ Autor } & Pais & Qtd Pub \\
\hline Del PretteA mir & BR & 34 \\
\hline Nordentoft M & DK & 33 \\
\hline Zigler Edwad & US & 31 \\
\hline Merrell Kenneth W & US & 30 \\
\hline Burns T & UK & 29 \\
\hline Rubin Kenneth H & DK & 27 \\
\hline Johnson S & UK & 27 \\
\hline Denham Susanne A & US & 26 \\
\hline DrakeRE & US & 26 \\
\hline Segrin Chris & US & 25 \\
\hline
\end{tabular}

\begin{tabular}{|l|l|r|}
\hline \multicolumn{1}{|c|}{ Autor } & Pais & QtdPub \\
\hline Fabes Richard A & US & 24 \\
\hline Penn David L & US & 23 \\
\hline Priebe S & UK & 23 \\
\hline Cartledge Gwendolyn & US & 23 \\
\hline Walker Hill M & US & 23 \\
\hline Liberman Robert P & US & 23 \\
\hline Jeppesen P & DK & 22 \\
\hline Vaughn Sharon & US & 22 \\
\hline Odom Samuel L & US & 21 \\
\hline Spence Susan H & AU & 21 \\
\hline
\end{tabular}

\begin{tabular}{|l|l|r|}
\hline \multicolumn{1}{|c|}{ Autor } & Pais & QtdPub \\
\hline Howes Carollee & US & 21 \\
\hline Botvin Gilbert J & US & 20 \\
\hline Elias MauriceJ & US & 20 \\
\hline Frankel Fred & US & 20 \\
\hline McGrew JH & US & 20 \\
\hline Riggio Ronald E & US & 20 \\
\hline Salyer SMP & US & 20 \\
\hline
\end{tabular}

Fonte: A autora (2014).

De outros países, encontram-se autores representativos do Brasil, como Z.A.P. Del Prette e Almir Del Prette; do Reino Unido, T. Burns, S, Johnson e S. Priebe; da Dinamarca, M. Nordentoft, K.H. Rubin e P. Jeppersen; da Austrália, Susan H. Spence. É importante enfatizar que o número de publicações de um autor não representa necessariamente o fator de impacto do mesmo. O fator de impacto baseia-se no índice de citações, o qual não foi coletado nesta pesquisa. Ainda assim, os autores que mais publicam em um campo de conhecimento são representativos, especialmente quando se trata de artigos científicos publicados em bases de dados internacionais com critérios rígidos de aceite.

\subsection{Publicações em HS por Assunto}

Os indicadores bibliométricos de publicações em HS por assunto (Tabela 2) são provenientes dos termos-chave originais encontrados nos próprios artigos, sem nenhuma indexação ou tratamento dos termos durante esta pesquisa, mostrando o que os próprios autores e indexadores das bases de dados definiram como termos-chave para os artigos.

O termo-chave mais frequente é "social skills" (7.471), seguido por outros bem menos frequentes: "interpersonal relations" (2.369), "social competence" (2.140), "empathy" (2.035). O termo "humanos" (1.591) é um qualificador usado comumente para diferenciar pesquisas com humanos de pesquisas animais nas BDLTCs, por isso é tão frequente.

Tabela 2 : Ranking dos primeiros 40 artigos sobre Habilidades Sociais por Assunto no período de 1912 a 2014.

\begin{tabular}{|l|r|}
\hline \multicolumn{1}{|c|}{ Assunto } & QtdPub \\
\hline $\begin{array}{l}\text { social skills } \\
\text { interpersonal } \\
\text { relations }\end{array}$ & 7.471 \\
\hline $\begin{array}{l}\text { social } \\
\text { competence }\end{array}$ & 2.369 \\
\hline empathy & 2.140 \\
\hline humanos & 1.035 \\
\hline $\begin{array}{l}\text { assertividade } \\
\text { assertiveness }\end{array}$ & 1.291 \\
\hline $\begin{array}{l}\text { social } \\
\text { interaction }\end{array}$ & 1.200 \\
\hline children & 1.185 \\
\hline $\begin{array}{l}\text { social skills } \\
\text { training }\end{array}$ & 889 \\
\hline
\end{tabular}

\begin{tabular}{|l|r|}
\hline \multicolumn{1}{|c|}{ Assunto } & QtdPub \\
\hline feminino & 862 \\
\hline humans & 813 \\
\hline masculino & 748 \\
\hline article & 708 \\
\hline male & 670 \\
\hline adulto & 635 \\
\hline female & 619 \\
\hline $\begin{array}{l}\text { assertiveness } \\
\text { (psychology) }\end{array}$ & 556 \\
\hline rela & 542 \\
\hline social behavior & 386 \\
\hline
\end{tabular}

\begin{tabular}{|l|r|}
\hline \multicolumn{1}{|c|}{ Assunto } & QtdPub \\
\hline $\begin{array}{l}\text { descriptive } \\
\text { statistics }\end{array}$ & 384 \\
\hline $\begin{array}{l}\text { human } \\
\text { questionnai } \\
\text { res }\end{array}$ & 376 \\
\hline $\begin{array}{l}\text { social } \\
\text { desirability }\end{array}$ & 353 \\
\hline adult & 351 \\
\hline psychology & 343 \\
\hline meia-idade & 331 \\
\hline child & 322 \\
\hline study & 321 \\
\hline autism & 316 \\
\hline
\end{tabular}

\begin{tabular}{|l|r|}
\hline \multicolumn{1}{|c|}{ Assunto } & QtdPub \\
\hline $\begin{array}{l}\text { college } \\
\text { students }\end{array}$ & 315 \\
\hline $\begin{array}{l}\text { research -- } \\
\text { finance }\end{array}$ & 303 \\
\hline adolescente & 280 \\
\hline $\begin{array}{l}\text { research } \\
\text { adolescent }\end{array}$ & 275 \\
\hline schizophrenia & 264 \\
\hline servi & 263 \\
\hline $\begin{array}{l}\text { assertiveness } \\
\text { training }\end{array}$ & 260 \\
\hline adolescents & 256 \\
\hline communication & 253 \\
\hline
\end{tabular}

\begin{tabular}{|l|r|}
\hline \multicolumn{1}{|c|}{ Assunto } & QtdPub \\
\hline personality & 250 \\
\hline aggression & 235 \\
\hline cognition & 226 \\
\hline $\begin{array}{l}\text { analysis of } \\
\text { variance }\end{array}$ & 225 \\
\hline socialization & 222 \\
\hline united states & 220 \\
\hline anxiety & 212 \\
\hline friendship & 208 \\
\hline social support & 202 \\
\hline education & 199 \\
\hline
\end{tabular}

Fonte: A autora (2014). 
O termo "assertividade", em português ou espanhol, aparece com maior frequência que o termo "assertiveness" (1.200), em inglês, possivelmente indicando que há um grande número de pesquisas sobre este tema nos países de língua portuguesa e espanhola. O termo "assertiveness (psychology)" (556) indica que pode haver outros escopos e campos de pesquisa, além da Psicologia que tratam deste tema. Diversos outros termos em português e espanhol reafirmam este achado.

Também são relevantes termos como "children" (1.137), "adulto" (635), "meia-idade" (331), "child" (322), "college students" (315), "adolescente” (280), "adolescent" (264), refletindo os públicos-alvo ou fases do desenvolvimento de que trata o CHS.

Outros termos-chave dizem respeito ao método, técnica ou procedimento utilizado, tais como "descriptive statistics" (384), "questionnaires" (370), "study" (321), "research" (275), "analysis of variance" (225), indicando uma boa quantidade de pesquisas descritivas, uso de questionários e de técnicas de análise de variância.

\subsection{Publicações em HS por Periódicos}

\section{Os indicadores bibliométricos de publicações em HS por periódico (}

Tabela 3) apresentam o ranking dos 40 periódicos que mais publicam sobre HS, destacando-se dezesseis deles com mais de 100 artigos.

Tabela 3 - Ranking dos primeiros 40 Artigos sobre Habilidades Sociais por Periódico no período de 1912 a 2014.

\begin{tabular}{|c|c|c|c|}
\hline Periódico & Qtd Publ & Periódico & Qtd Publ \\
\hline Journal of Personality and Social Psychology & 309 & Journal of Clinical Child Psychology & 85 \\
\hline Psychological Reports & 287 & Adolescence & 83 \\
\hline Journal of Autism and Developmental Disorders & 271 & American Journal of Mental Deficiency & 82 \\
\hline Child Development & 248 & Journal of Psychology: Interdisciplinary and Applied & 80 \\
\hline Journal of Consulting and Clinical Psychology & 194 & Journal of Applied Behavior Analysis & 80 \\
\hline Developmental Psychology & 183 & Research in Developmental Disabilitie & 79 \\
\hline Behavior Therapy & 167 & The American Journal of Psychiatry & 78 \\
\hline Journal of Clinical Psychology & 165 & Education and Treatment of Children (ETC) & 78 \\
\hline Behavior Modification & 146 & Behavioral Disor ders & 77 \\
\hline Journal of Child Psychology and Psychiatry and Allied Disciplines & 131 & Journal of Learning Disabilties & 76 \\
\hline Behariour Research and Ther apy & 124 & Journal of Youth and Adolescence & 75 \\
\hline Journal of Abnormal Child Psychology & 123 & Journal of Personality & 73 \\
\hline PsYcCRITIQUES & 123 & Sex Roles & 72 \\
\hline Social Development & 116 & Early Child Development andCare & 70 \\
\hline Personality and Social Psychology Bulletin & 108 & Autism: The International Journal of Research and Pr actice & 70 \\
\hline Journal of Behavior Therapy and Experimental Psychiatry & 107 & Schizophrenia Research & 70 \\
\hline The Journal of Social Psychology & 95 & Journal of Advanced Nursing & 69 \\
\hline Psychology in the Schools & 95 & School Psychology Review & 69 \\
\hline Personality and Ind i idual Differences & 91 & Journal of Applied Developmental Psychology & 67 \\
\hline Journal of Counseling Psychology & 89 & Journal of the American Academy of Child and Adolescent Psychiatry & 67 \\
\hline
\end{tabular}

Fonte: A autora (2014).

Os primeiros do ranking são: Journal of Personality and Social Psychology (309); Psychological Reports (287); Journal of Autism and Developmental Disorders (271); Child Development (248). O primeiro destes, com foco em Psicologia Social e Personalidade, contém diversos artigos sobre assertividade, agressividade, motivação, ansiedade, entre outros. O segundo, com foco mais geral e o terceiro relacionado a autismo e transtornos do desenvolvimento; o quarto do ranking, voltado ao desenvolvimento infantil.

Os principais temas destes periódicos são respectivamente: Criança; Psicologia do Desenvolvimento; Psicologia Social; Terapia Comportamental; Psiquiatria; Psicologia; Adolescente; Personalidade; Transtornos Mentais; Autismo; Transtornos do Desenvolvimento; Psicologia Clínica; Psicologia Escolar; Papel Sexual; Enfermagem; Educação; Transtornos Comportamentais. Os assuntos variam, mas há uma prevalência de publicações relativas ao tema da criança, do desenvolvimento e dos transtornos. 


\section{Conclusões}

Esta pesquisa teve por objetivo contribuir para a sistematização do conhecimento do CHS por meio de uma análise bibliométrica do campo, sustentada por um corpus extraído da literatura internacional sobre HS (Corpus HS), utilizando recursos da Ciência da Informação e da Ciência da Computação.

O corpus foi tratado e disponibilizado para acesso on-line e gratuito e para atualização contínua dos metadados das publicações. O corpus foi armazenado em base MySQ possibilitando o desenvolvimento de indicadores bibliométricos, que consistem em uma série de estatísticas, com sumarização de dados, na forma de gráficos e tabelas relativas a aspectos do $\mathrm{CHS}$.

Os indicadores bibliométricos do CHS mostram um crescimento representativo da produção sobre o tema das habilidades sociais a partir da década de 1970, com crescimento exponencial do campo a partir dos anos 2000. A pesquisa em HS é conduzida principalmente por pesquisadores dos Estados Unidos, Brasil, Reino Unido e Dinamarca. Os principais assuntos em HS são "social skills", "interpersonal relations", "social competence", "empathy", "assertividade", "assertiveness", "children", "adulto", "college students", entre outros. Os principais periódicos que publicam HS são: Journal of Personality and Social Psychology; Psychological Reports; Journal of Autism and Developmental Disorders e Child Development, sendo seus principais temas: Criança; Psicologia do Desenvolvimento; Psicologia Social; Terapia Comportamental; e outros.

Com os resultados desta pesquisa, espera-se que o CHS tenha alguns subsídios para alavancar seu crescimento e expansão, com base em indicadores dinâmicos que contribuam com a organização e sistematização do campo, de modo que este cumpra seu objetivo como disciplina do saber. Como exemplo de aplicação, os indicadores relativos a publicações de autores em determinados países poderiam servir como instrumentos para obtenção de fundos de pesquisa: de um lado, para instigar os países que estão produzindo pouco a produzir mais; de outro lado, para ampliar e aprofundar a pesquisa nos países que já a produzem. Outro exemplo de indicadores relativos a periódicos mostram uma maior frequência de artigos sobre habilidades sociais em crianças, sugerindo a possibilidade de estudos com outras faixas etárias, o que poderia justificar o fomento à pesquisa de habilidades sociais de adolescentes, adultos e idosos.

Uma limitação da pesquisa foi que não se encontrou uma ferramenta de livre acesso para extração do corpus de todas as bases, o que dificultou o processo, diferente para cada uma delas. Espera-se que em breve a comunidade científica e tecnológica do campo da Computação providencie este tipo de ferramenta como software livre, facilitando os processos de extração de corpus, que podem ser úteis para diversas finalidades, além de análises cientométricas e bibliométricas.

Como perspectiva em relação à distribuição de HS por países, recomenda-se uma pesquisa que identifique os países de origem das publicações, o que não foi possível neste estudo, pois tal informação não se encontrava disponível na maioria dos metadados. Este tipo de pesquisa possibilitaria compreender o que as comunidades de pesquisadores estão estudando nos países mais representativos em estudos sobre a HS. Recomenda-se a análise de outros tipos de indicadores, tais como índices de autoria, taxas de colaboração e identificação de grupos disciplinares dentro da grande área do CHS. 


\section{Referências}

Argyle, M. (1978). Psicología del comportamiento interpersonal. Madrid: Alianza Editorial.

Bandura, A. (1986). Social foundations of thought and action: a social cognitive theory. New Jersey: Prentice Hall.

Bolsoni-Silva, A. T., Del Prette, Z. A., Del Prette, G., Montanher, A. R. P., Bandeira, M., Del Prette, A., ... Del Prette, A. (2006). A área das habilidades sociais no Brasil: uma análise dos estudos publicados em periódicos. Estudos sobre habilidades sociais e relacionamento interpessoal, 17-45.

Caballo, V. E. (2005). Manual de evaluación y entrenamiento de las habilidades sociales. Siglo XXI de España Editores.

CAPES. (2010). Portal .periodicos. CAPES. 2010. Recuperado 4 de julho de 2011, de

http://www.periodicos.capes.gov.br.ez31.periodicos.capes.gov.br/index.php?option=com pmetabusca\&mn=70\&smn=78\&base $=$ find $-\mathrm{db}-1 \&$ type $=\mathrm{b} \& \mathrm{mn}=88 \& \mathrm{smn}=91$.

Costa, T., Lopes, S., \& Fernández-Llimós, F. (2012). A bibliometria e a avaliação da produção científica: indicadores e ferramentas. Recuperado de http://comum.rcaap.pt/handle/123456789/4620

Del Prette, A. (1998). Efeitos de uma intervenção sobre a topografia das habilidades sociais de professores. Psicologia Escolar e Educacional, 2(1), 11-22.

Del Prette, A., \& Del Prette, Z. A. P. (2010). Habilidades sociais e Análise do Comportamento: Proximidade histórica e atualidades. Revista Perspectivas, 1(02), 104-115.

Del Prette, A., \& Del Prette, Z. A. P. (Orgs.). (2011). Habilidades sociais: intervenções efetivas em grupo. São Paulo: Casa do Psicólogo.

Del Prette, Z. A. P., \& Del Prette, A. (1999). Psicologia das habilidades sociais: terapia, educação e trabalho. Petrópolis: Vozes.

Del Prette, Z. A. P., Del Prette, A., Haase, V. G., ROTHE-NEVES, R., KÄPPLER, C., Teodoro, M. L. M., \& Wood, G. M. O. (2000). Treinamento em habilidades sociais: Panorama geral da área. In Psicologia do desenvolvimento: Contribuições interdisciplinares (p. 249-264).

Fumo, V. M. ., Manolio, C. L., Bello, S., \& Hayashi, M. C. P. . (2009). Produção científica em habilidades sociais: Estudo bibliométrico. Revista Brasileira de Terapia Comportamental e Cognitiva, 11(2), 246-266.

Gault, R. H. (1912). City Children and Crime. Journal of the American Institute of Criminal Law and Criminology, 3(4), 499-501.

Gresham, F. M. (2009). Análise do comportamento aplicada às habilidades sociais. In Z. A. P. Del Prette \& A. Del Prette (Orgs.), Psicologia das habilidades sociais: diversidade teórica e suas implicações (p. 17-66). Petrópolis: Vozes.

Hargie, O. D., Saunders, C., \& Dickson, D. (1994). Social skills in interpersonal communication. Chesham: Psychology Press Recuperado de http://books.google.com.br/books?hl=pt-BR\&lr=\&id=vdH8vjQvM-

MC\&oi=fnd\&pg =PR9\&dq=Hargie, + Saunders+\%26+Dickson\&ots=nxz6OCdrCF\&sig=30oXOliu2zuRyqUQkYy-4k6-|3w

McFall, R. M. (1982). A review and reformulation of the concept of social skills. Behavioral assessment, 4, 1-33.

Mugnaini, R. (2006). Caminhos para adequação da avaliação da produção científica brasileira: impacto nacional versus internacional (Tese de Doutorado). USP/ECA, São Paulo. Recuperado de http://www.teses.usp.br/teses/disponiveis/27/27151/tde-11052007-091052/en.php.

Peña, L. J. M. (2012). Análisis bibliométrico sobre la producción científica archivística en la Red de Revistas Científicas de América Latina y el Caribe (Redalyc) durante el período 2001-2011. Biblios, (48), 1-11.

Silva, J. A., \& Bianchi, M. L. P. (2001). Cientometria: a métrica da ciência; Scientometrics: the measurement of science Paidéia, 11(21), 5-10.

Skinner, B. F. (1953). Science and human behavior. New York: The Free Press.

Spinak, E. (1998). Indicadores cienciométricos. Ci. Inf, 27(2), 141-148.

Trower, P. (1995). Adult social skills: state of the art and future directions. Handbook of psychological skills training: clinical techniques and applications, 54-80. 


\section{Dados da autora}

Eliane Colepícolo

Doutora em Psicologia pela UFSCar, Mestre em Informática em Saúde pela Universidade Federal de São Paulo - UNIFESP/EPM e graduada em Ciência da Informação e Biblioteconomia pela Universidade Federal de São Carlos - UFSCar (1999), desde 2008 é Chefe da Seção de Acesso a Bases de Dados da Biblioteca Comunitária da UFSCar e pesquisadora interdisciplinar especialista em Estudos Bibliométricos e Cientométricos para avaliação de Áreas do Conhecimento e Campos de Pesquisa, tais como o Campo das Habilidades Sociais e a Informática em Saúde. Tem experiência em Ciências da Informação e Computação.

ecolepicolo@hotmail.com

Recebido - Received : 2014-08-26

Aceitado - Accepted : 2014-12-30

\section{(cc) BY}

This work is licensed under a Creative Commons Attribution 4.0

United States License.

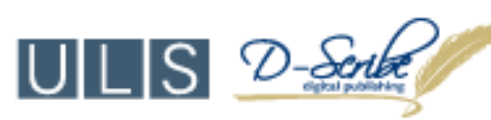

This journal is published by the University Library System of the University of Pittsburgh as part of its D-Scribe Digital Publishing Program and is cosponsored by the University of Pittsburgh Press. 